\title{
SILYBUM MARIANUM E SUAS ATIVIDADES FARMACOLÓGICAS: UMA REVISÃO INTEGRATIVA
}

\author{
SILYBUM MARIANUM AND ITS PHARMACOLOGICAL ACTIVITIES: AN \\ INTEGRATIVE REVIEW
}

\author{
Antonio Rafael da Silva ${ }^{1}$, Maria Nathalya Costa de Souza ${ }^{2}$ \\ Universidade Regional do Cariri- URCA ${ }^{1}$, Faculdade de Medicina Estácio de Juazeiro do Norte-CE ${ }^{2}$
}

\begin{abstract}
The aim of this study is to verify the main pharmacological properties of Silybum marianum. This is an integrative review study, having as inclusion criteria: articles in English, Portuguese and Spanish in the following databases: Virtual Health Library (BVS), (PUBMED) and Scientific Electronic Library Online (SCIELO), using the descriptors: Silimarina, Cardo Mariano, Silybum marianum. This research took place from March to September 2020, where 8 scientific articles that met the inclusion criteria published in the period 2010 to June 2020 were selected. The exclusion criteria were: review articles, articles that did not meet the theme, book chapters, dissertations, non-scientific texts and scientific articles without availability of the full text. The selected literature presented several pharmacological properties of silymarin, ranging from its antioxidant capacity to the prevention of mucositis in patients with head and neck cancer, in addition to hepatoprotective and antidiabetic properties. It was observed that the treatment with silymarin and its constituents are able to attenuate common diabetic complications in several organs, as well as emphasizing the antioxidant, anti-inflammatory, dose-dependent anti-apoptotic and cellular transport modifier properties present in this plant, hence silymarin it has important therapeutic properties for the most diverse ailments and further in-depth studies are needed against these properties. It was soon also seen that there is a paucity of research on domestication and investigation of the pharmacological properties of this plant.
\end{abstract}

Keywords: Silimarina marianum; Milk thistle; Silymarin.

\section{Resumo}

O objetivo desse estudo consiste em verificar as principais propriedades farmacológicas da Silybummarianum. Trata-se de um estudo de revisão integrativa, tendo como critérios de inclusão: artigos nos idiomas inglês, português e espanhol nas seguintes bases de dados: Biblioteca Virtual de Saúde (BVS), (PUBMED) e Scientific Eletronic Library Online (SCIELO), utilizando os descritores: Silimarina, Cardo-Mariano, Silybummarianum. Silimarinamarianum; Milkthistle; Silymarin. Essa pesquisa ocorreu nos meses de março a setembro de 2020 onde foram selecionados 8 artigos científicos que enquadraram se aos critérios de inclusão publicados no período de 2010 a junho de 2020. Os critérios de exclusão estavam: artigos de revisões, artigos que não se enquadravam ao tema, capítulos de livros, dissertações, textos não científicos e artigos científicos sem disponibilidade do texto na integra. A literatura selecionada apresentou diversas propriedades farmacológicas da silimarina que vão desde a capacidade antioxidante até a prevenção da mucosite em pacientes com câncer de cabeça $e$ pescoço, além de propriedades hepatoprotetoras $e$ antidiabéticas. Observou-se que o tratamento com silimarina e os seus constituintes são capazes de atenuar complicações diabéticas comuns em vários órgãos, bem como enfatizaram as propriedades antioxidantes, anti-inflamatórios, antiapoptóticos dose-dependentes e modificadores de transportadores celulares presentes nessa planta, logo a silimarina apresenta importantes propriedades terapêuticas para as mais diversas enfermidades sendo necessários novos estudos aprofundados frente a essas propriedades. Logo foi visto também que há uma escassez de pesquisas sobre a domesticação $e$ investigação das propriedades farmacológicas desta planta.

Palavras-chave: Silimarina, Cardo-Mariano, Silybummarianum. 
A fitoterapia consiste na utilização de plantas medicinais em distintas formas farmacêuticas para solucionar ou auxiliar no tratamento de enfermidades. Estas passam por processos técnicos farmacêuticos até chegar no produto final e se tornarem o medicamento fitoterápico. Os medicamentos fitoterápicos são produtos elaborados a partir de substâncias vegetais ativas que oferecem a resolução para determinadas doenças. ${ }^{1,2}$

O Brasil é um país rico em biodiversidade, com um arsenal de plantas a serem estudadas, onde essas podem trazer diversos benefícios a saúde, quando utilizadas de forma correta para o tratamento das mais variadas doenças, logo se fazem necessárias novas pesquisas frente aos potenciais farmacológicos dessas plantas brasileiras. ${ }^{3}$

Silybummarianum,

conhecida popularmente como silimarina ou cardo de leite, é uma planta da família Asteraceae. É originária do sul da Europa até a Ásia e no momento atual encontrada em todo o mundo. Na alopatia e na fitoterapia, é administrada em doenças hepáticas (icterícia, cirrose e hepatite), em doenças da vesícula biliar e possui função hepatoprotetora contra venenos. ${ }^{4}$

O extrato dessa planta tem seu uso medicinal sob o nome de silimarina, que é composta por um grupo com várias substâncias e tem como um dos seus principais constituintes: os flavonoides, que são capazes de proporcionar proteção a planta bem como apresentam importantes propriedades farmacológicas, dentre essas: propriedades antitumorais, antiinflamatórias, antivirais, antioxidantes, dentre outras. $^{5}$

Os mecanismos de ação da Silybummarianumrelacionados a sua ação hepatoprotetora, estão associados as atividades antioxidantes e sequestro de radicais livres, que são capazes de estimular processos regenerativos, reguladores e estabilizadores da membrana celular. Essas ações ocorrem a partir da inibição da deposição de fibras de colágeno, do aumento da concentração de glutationa celular e estimulação da DNA polimerase. ${ }^{6}$

Diante disso, o objetivo desse estudo consiste em realizar uma revisão integrativa para avaliar as principais propriedades farmacológicas e segurança da Silybummarianum.

\section{Delineamento do estudo}

Uma revisão integrativa foi conduzida para compor uma amostra de estudos com a Silybummarianum. A revisão integrativa inclui a análise de pesquisas relevantes que dão suporte para a tomada de decisão e a melhoria da prática clínica. Ela possibilita a síntese do estado do conhecimento de um determinado assunto, além de apontar lacunas do conhecimento que precisam ser preenchidas com a realização de novos estudos.

\section{Processo de aquisição de literatura}

Para iniciar a busca, o ponto de partida foi desenvolvido de acordo com a estratégia de busca PVO. Foram identificados os descritores em ciências saúde (DeCS): Silimarina, CardoMariano, Silybummarianum.Silymarin, MilkThistle, Silybummarianum.

\section{Fontes de informações}

As buscas ocorreram nos bancos de dados da Biblioteca Virtual de Saúde (BVS), (PUBMED) e Scientific Eletronic Library Online (SCIELO), que indexam as principais evidências nacionais e internacionais. O processo de elegibilidade ocorreu apenas por um pesquisador e a extração de dados deu-se através do desenvolvimento de uma matriz de evidências e foram extraídos os dados dos estudos: autor, ano da publicação, delineamento, amostra e desfechos. Os dados foram categorizados de acordo com os desfechos de indicações e efeitos já comprovados.

\section{Critérios de inclusão dos estudos e elegibilidade}

Os critérios de elegibilidade foram estabelecidos de acordo com o que propomos indagar na introdução: I) Indicações medicinais, II) Bioatividades comprovadas III) Estudos in vivo, IV) Estudos in vitro, V) Sem restrições de idiomas ou temporais. Os critérios de exclusão serão: a) Estudos secundários e b) estudos que não versam especificamente de indicações e efeitos da Silybummarianum. Que estivessem nos idiomas português e inglês, publicados no período de 2010 a junho de 2020. 
Figura 1 - Fluxograma do processo de seleção dos estudos da Silybummarianum.

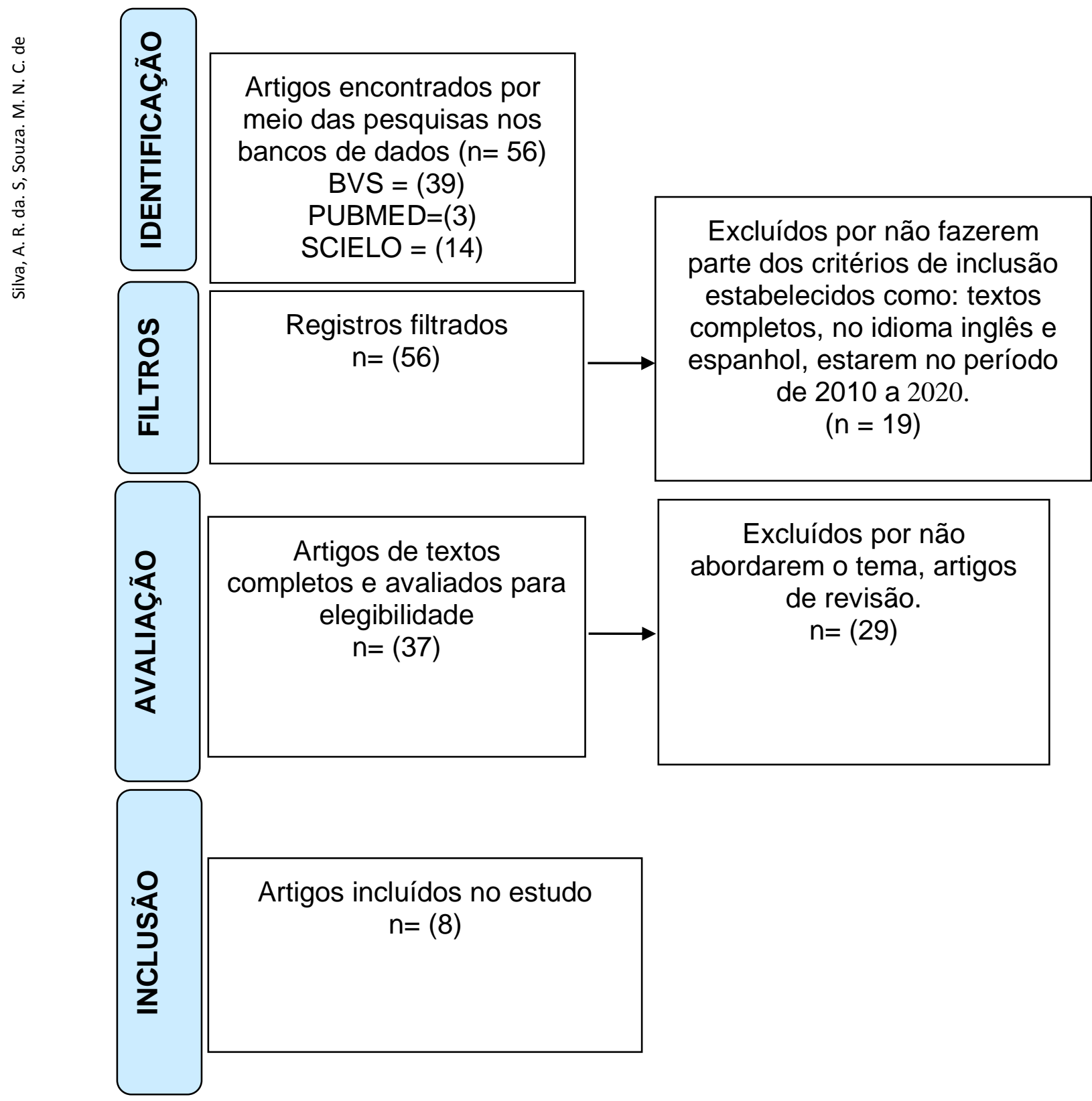


Os principais resultados encontrados assim como a identificação dos artigos/ autores $\stackrel{\infty}{\sim} \quad$ selecionados foram dispostos e organizados em um quadro, quadro 1.

Quadro 1 - Resultado da seleção de estudos.

\begin{tabular}{|c|c|c|c|}
\hline TíTULO & AUTOR/ ANO & OBJETIVO & $\begin{array}{c}\text { RESULTADOS } \\
\text { ENCONTRAD } \\
\text { OS }\end{array}$ \\
\hline $\begin{array}{l}\text { Silymarinadministrationduringpregnancyandbrea } \\
\text { stfeeding: } \\
\text { evaluationofinitialdevelopmentandadultbehavior } \\
\text { ofmice. }\end{array}$ & BARBOSA $^{7}$ & $\begin{array}{l}\text { Avaliar o } \\
\text { efeito do } \\
\text { tratamento } \\
\text { com silimarina } \\
\text { durante a } \\
\text { gravidez } \\
\text { eamamentaçã } \\
\text { o. }\end{array}$ & $\begin{array}{l}\text { Tratamento } \\
\text { de longo } \\
\text { prazo com } \\
\text { silimarina } \\
\text { durante a } \\
\text { gravidez e a } \\
\text { amamentaçã } \\
\text { o } \\
\text { não produziu } \\
\text { nenhum } \\
\text { efeito } \\
\text { significativo } \\
\text { no } \\
\text { comportamen } \\
\text { to ou } \\
\text { processos de } \\
\text { consolidação } \\
\text { da memória } \\
\text { na geração. }\end{array}$ \\
\hline $\begin{array}{l}\text { MilkThistleTreatmentofChildrenandAdultswithTri } \\
\text { chotillomania: A Double-Blind, Placebo- } \\
\text { Controlled, Cross-Over Negative Study }\end{array}$ & GRANT, et al. $^{8}$ & $\begin{array}{l}\text { O objetivo do } \\
\text { presente } \\
\text { estudo era } \\
\text { determinar a } \\
\text { eficácia e } \\
\text { tolerabilidade } \\
\text { da silimarina } \\
\text { em crianças e } \\
\text { adultos com }\end{array}$ & $\begin{array}{l}\text { Os resultados } \\
\text { mostraram } \\
\text { que o cardo } \\
\text { leiteiro tem } \\
\text { melhorias } \\
\text { significativas } \\
\text { para o } \\
\text { tratamento } \\
\text { de }\end{array}$ \\
\hline
\end{tabular}


Continuação.

$\stackrel{\stackrel{\sim}{N}}{\sim}$

\begin{tabular}{|c|c|c|c|}
\hline & & tricotilomania. & tricotilomania \\
\hline 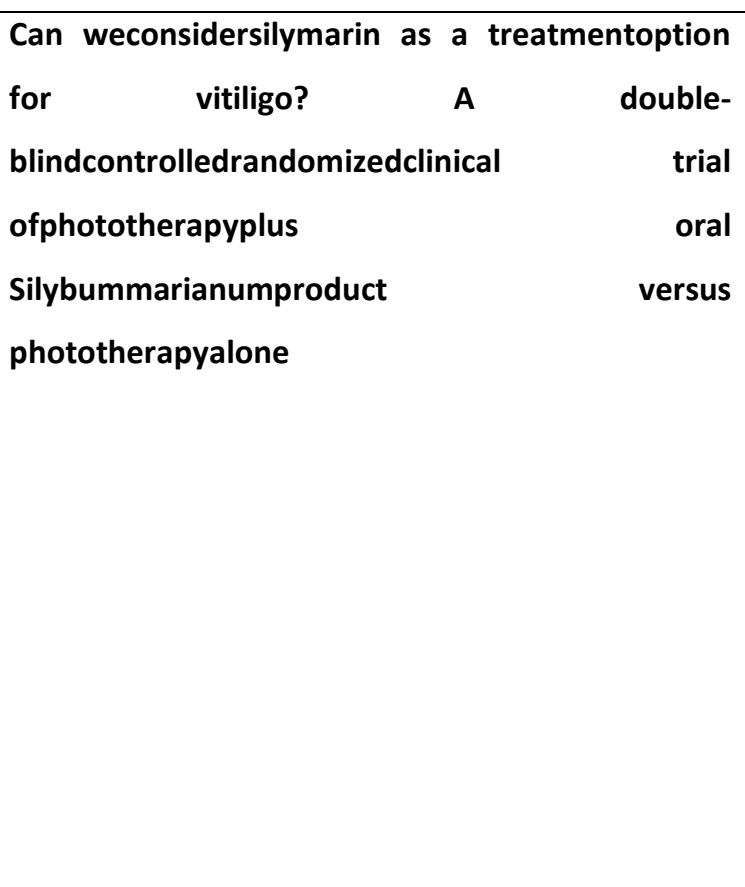 & $\mathrm{JOWKAR}^{9}$ & $\begin{array}{l}\text { Mostrar o } \\
\text { efeito da } \\
\text { silimarina oral } \\
\text { para esse } \\
\text { tratamento. }\end{array}$ & $\begin{array}{l}\text { O estudo } \\
\text { mostrou que } \\
34 \text { de } 40 \\
\text { pacientes } \\
\text { tiveram bons } \\
\text { resultados a } \\
\text { com } \\
\text { silimarina } \\
\text { como } \\
\text { alternativa } \\
\text { para } \\
\text { tratamento } \\
\text { desses } \\
\text { pacientes. }\end{array}$ \\
\hline $\begin{array}{l}\text { Lowerglycemicindicesandlipid profile amongtype } \\
2 \text { diabetes mellitus patientswhoreceived novel } \\
\text { dose ofSilybummarianum (L.) Gaertn. (silymarin) } \\
\text { extractsupplement: A A Ariple- } \\
\text { blindedrandomizedcontrolledclinicaltrial }\end{array}$ & KOUJAN et al. ${ }^{10}$ & $\begin{array}{l}\text { Avaliar os } \\
\text { efeitos da } \\
\text { silimarina nos } \\
\text { índices } \\
\text { glicêmicos e } \\
\text { perfil lipídico } \\
\text { sérico em } \\
\text { diabetes } \\
\text { mellitus tipo } \\
2 .\end{array}$ & $\begin{array}{l}\text { Os resultados } \\
\text { mostraram o } \\
\text { potencial } \\
\text { antioxidante } \\
\text { e efeito } \\
\text { antiinflamató } \\
\text { rio da } \\
\text { silimarina em } \\
\text { pacientes } \\
\text { com D2M de } \\
\text { forma em que } \\
\text { evitou o } \\
\text { aumento da } \\
\text { glicose } \\
\text { plasmática e } \\
\text { pancreática } \\
\text { peroxidação } \\
\text { lipídica em } \\
\text { ratos com } \\
\text { diabetes, } \\
\text { melhorando } \\
\text { os índices }\end{array}$ \\
\hline
\end{tabular}




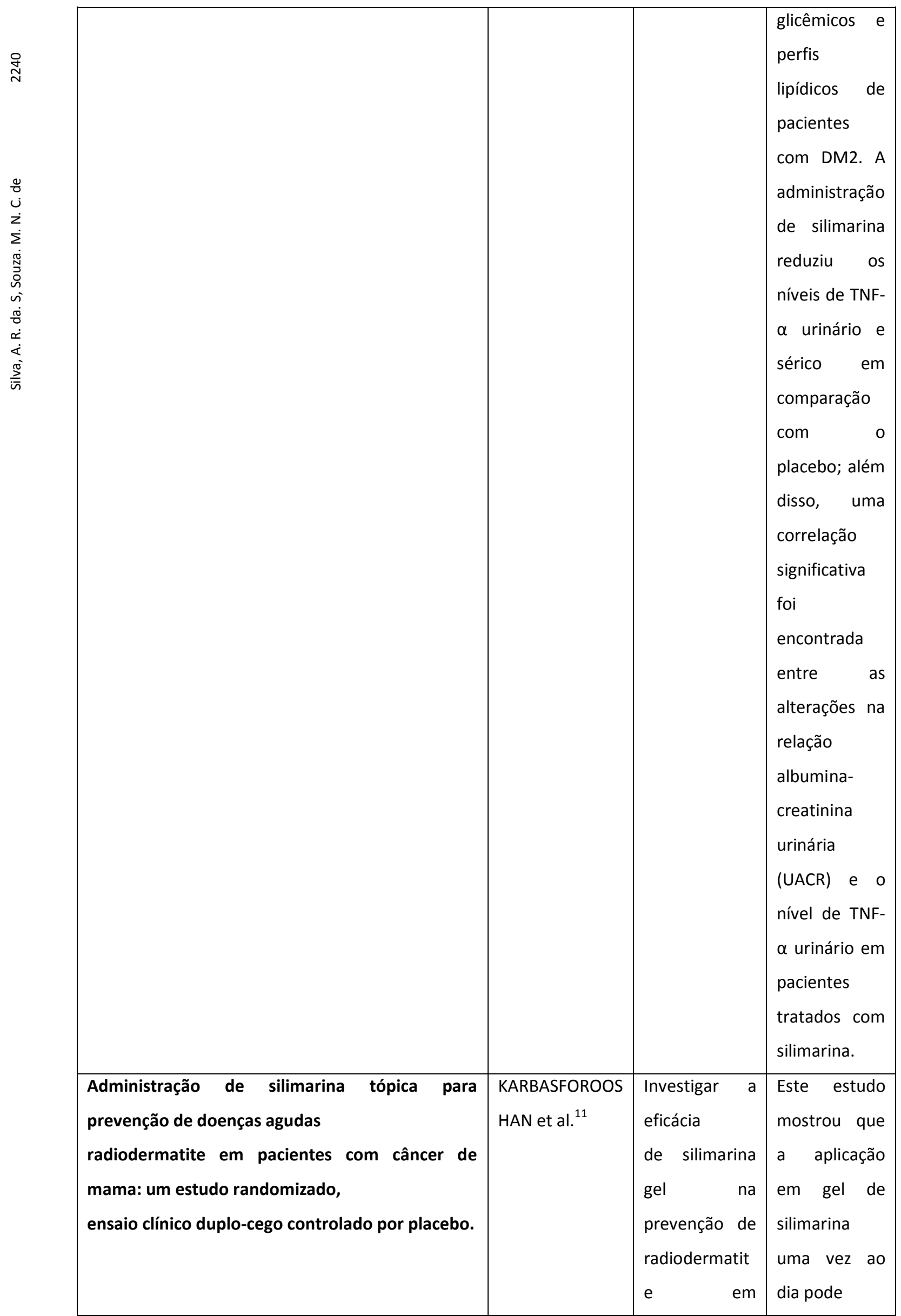




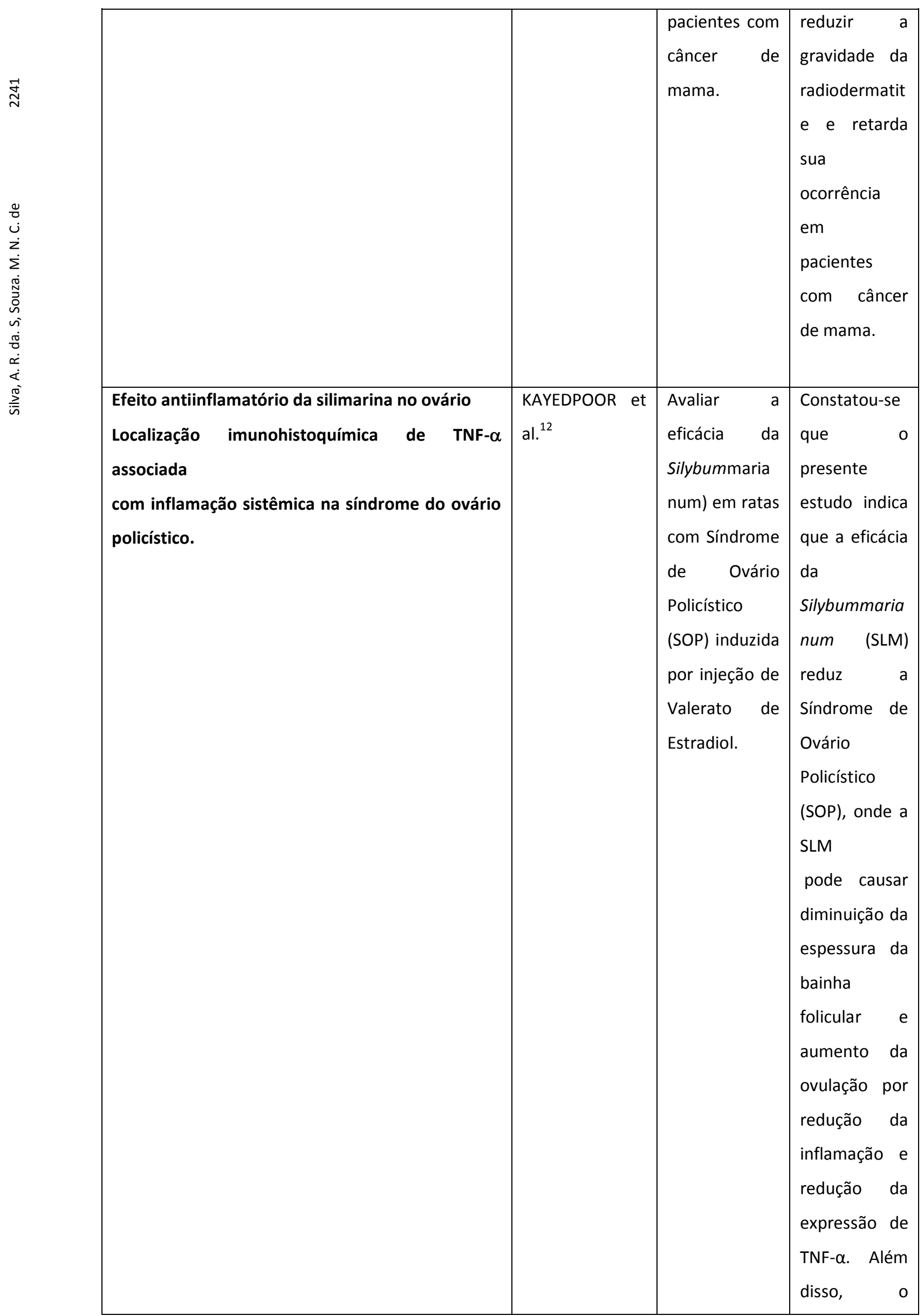


Continuação.

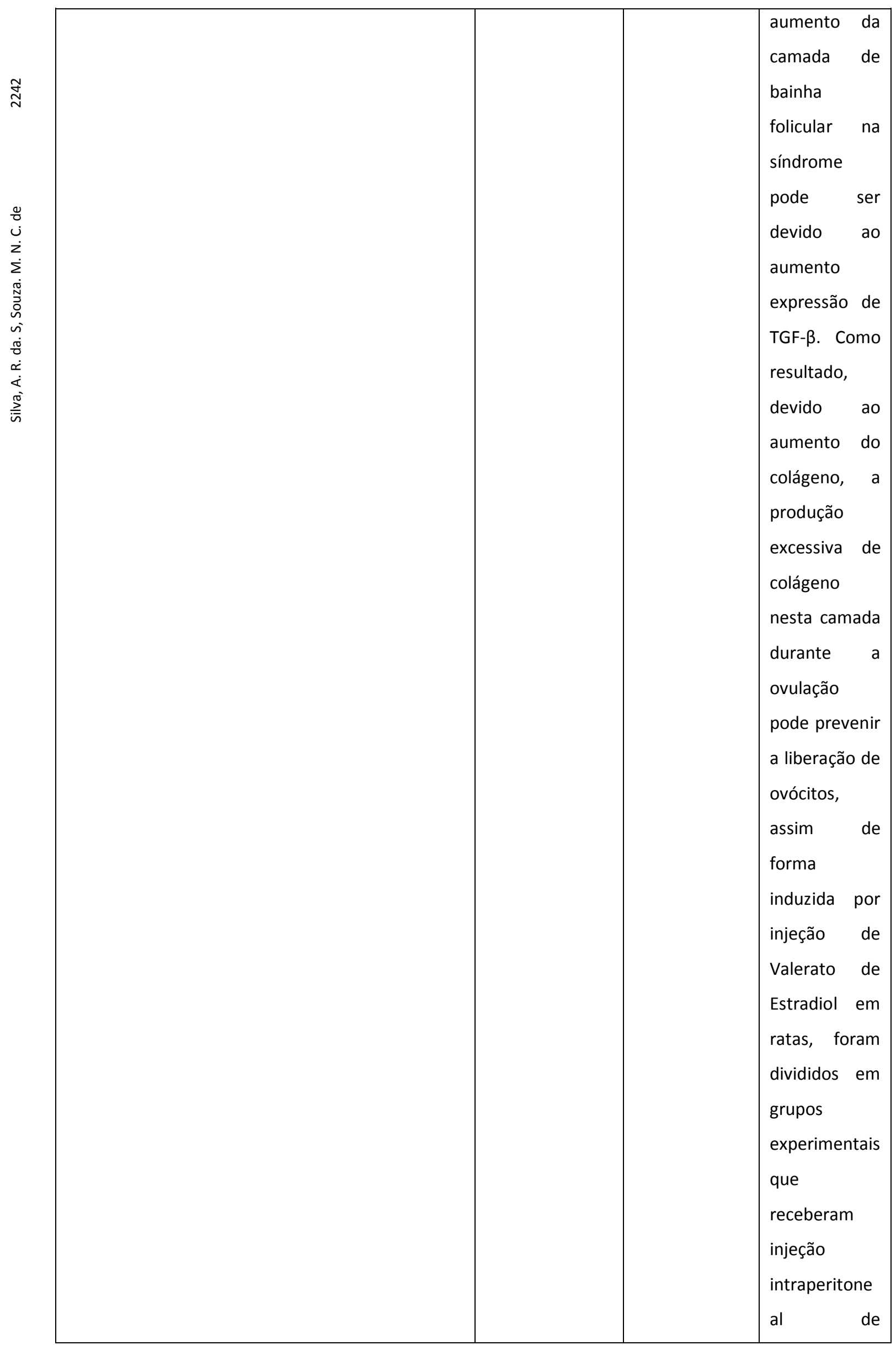




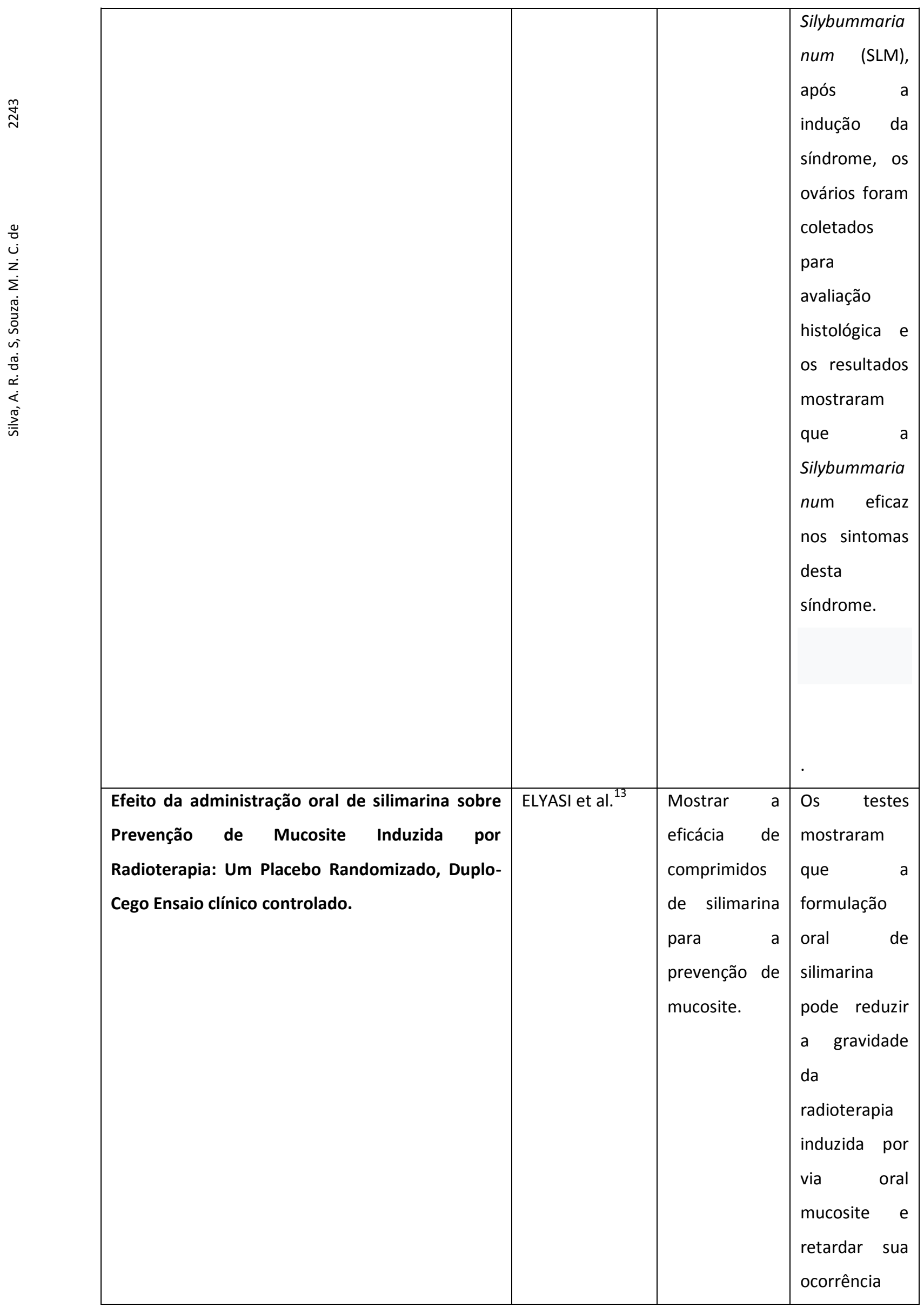




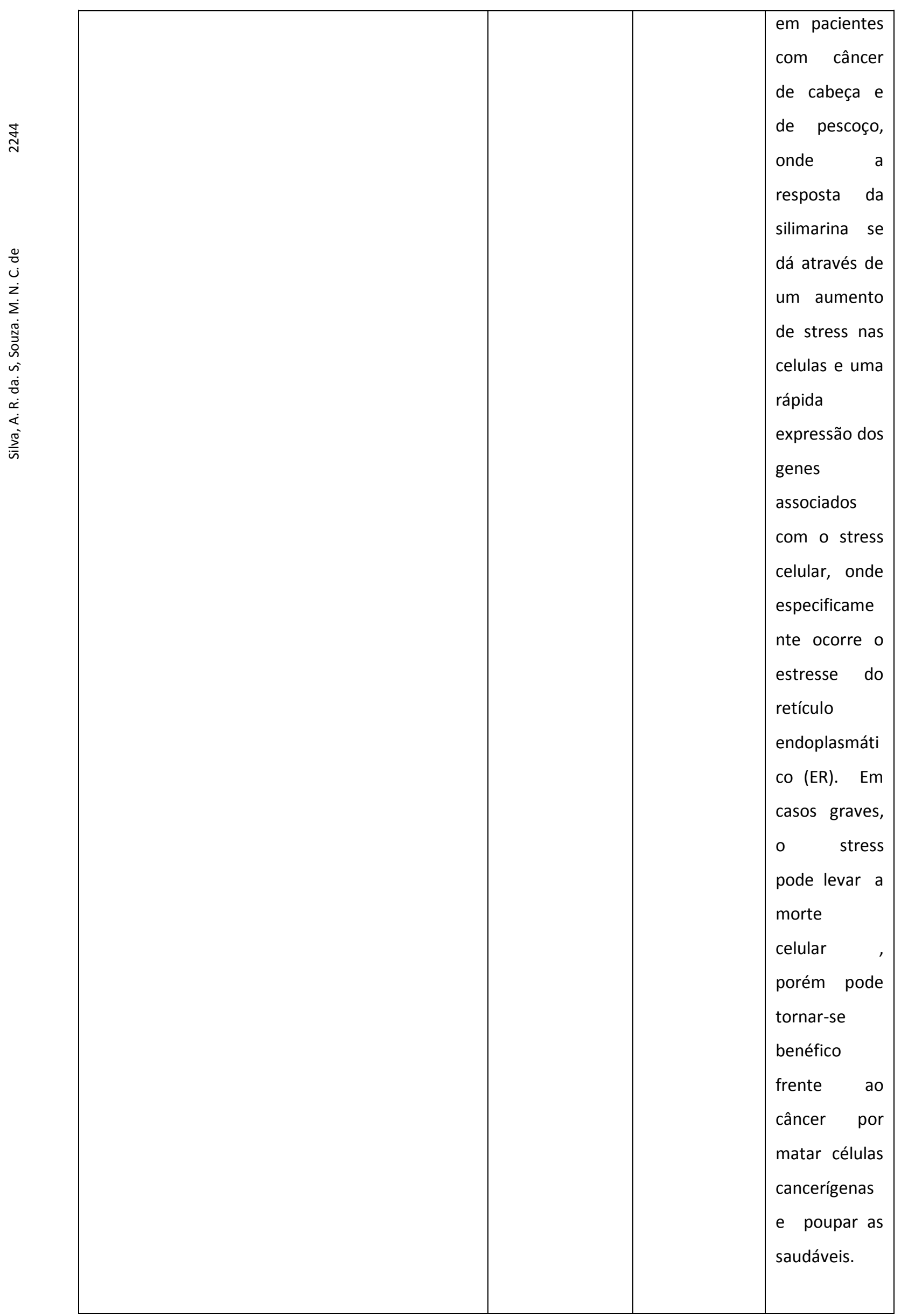




\begin{tabular}{|c|c|c|c|}
\hline $\begin{array}{l}\text { Efeito da silimarina nos rins de ratos que sofrem } \\
\text { de indução de aloxana } \\
\text { Diabetes mellitus }\end{array}$ & SOTO et al. ${ }^{14}$ & $\begin{array}{l}\text { Diminuir } \\
\text { significativam } \\
\text { ente o nível } \\
\text { de diabetes } \\
\text { em } \\
\text { grupo de ratos } \\
\text { diabéticos ao } \\
\text { longo do } \\
\text { experimento. }\end{array}$ & $\begin{array}{l}\text { A silimarina } \\
\text { bloqueou } \\
\text { diminuições } \\
\text { induzidas por } \\
\text { aloxana na } \\
\text { atividade e } \\
\text { alterações } \\
\text { nos níveis de } \\
\text { expressão } \\
\text { dessas } \\
\text { enzimas } \\
\text { antioxidantes } \\
\text { renais e } \\
\text { também } \\
\text { restauravam } \\
\text { a histologia } \\
\text { renal normal } \\
\text { em ratos } \\
\text { diabéticos. }\end{array}$ \\
\hline
\end{tabular}

Fonte: Dados da Pesquisa (2020).

A similarina tem seu uso terapêutico principalmente como um hepatoprotetor, porém essa também vem apresentando resultados promissores frente ao tratamento de várias doenças, como diabetes, Alzheimer, Parkinson, sepse, queimaduras, osteoporose, colestase, hipercolesterolemia e neoplasias, além da mesma possuir importantes propriedades antioxidantes. ${ }^{4}$

As propriedades antioxidantes da silimarina são capazes de reduzir espécies reativas de oxigênio, inibindo, portanto, danos celulares. Além de provocarem melhora no sistema antioxidante, observado em estudos experimentais, em decorrência ao aumento de enzimas como glutationa redutase, glutationa peroxidase, superóxido dismutase e catalase, todas com função antioxidante. ${ }^{10}$

Estudos já apontam efeitos benéficos da silimarina no tratamento do diabetes essa aumentou à expressão gênica da insulina no pâncreas, a neoformação de células, a insulina sérica e normalizou a glicemia quando realizado

testes em animais, avaliando o efeito da silimarina em um modelo de diabetes induzida pela estreptozotocina e nicotinamida, no qual os animais tratados tiveram redução da glicemia, volume urinário, albumina urinária, e da creatinina e ácido úrico séricos, comprovando os efeitos nefro protetores. ${ }^{4}$

De acordo com Kayedpoorno et al., ${ }^{12}$ seu estudo o TNF- $\alpha$ (Fator Nervoso Tumoral) que é uma expressão anormal que pode levar a condições patológicas, como inflamação e policístico, mostram que o grupo com SOP de 144 ratas adultas com a administração diariamente do extrato da silimarina por injeção intraperitoneal diminuiu a espessura da bainha folicular e a redução do SOP no ovário, observando assim a eficácia da Silimarina frente a essa síndrome. 
Com os estudos de Koujan et al. ${ }^{10}$, participantes foram atribuídos aleatoriamente ao uso da silimarina para avaliar o seu efeito nos índices glicêmicos e em perfil lipídicos em pacientes com diabetes tipo 2, sendo administrado $140 \mathrm{mg}$ três vezes ao dia por 45 dias, com isso mostrou bons resultados com as taxas reduzidas dos 40 pacientes que fizeram o uso da silimarina, em complemento a isso nas pesquisas de Soto et al. ${ }^{14}$, é observado que o estresse oxidativo contribui para o desenvolvimento de diabetes mellitus e o rins são sujeitos a causar danos por radicais livres, com isso foi testado o efeito da silimarina como um eliminador de radicais livres, onde foi visto que a silimarina evitou danos aos tecidos e assim conforme descrito por ambos os autores essa consegue reduzir os índices glicêmicos e em decorrência da sua capacidade de eliminar radicais livres pode ser considerada potencialmente no tratamento de nefropatia diabética.

Karbasforooshan et al., ${ }^{11}$ apresentaram em seu estudo que na radioterapia a dermatite por radiação é comum e a silimarina em forma de gel teria efeito preventivo para esses pacientes utilizando uma vez ao dia, começando no primeiro dia de radioterapia, com isto foi observado que após 5 semanas da administração do gel de silimarina reduziu a gravidade radiodermatite, diante disso pesquisas de Elyasi et al., ${ }^{13}$ constataram que a silymarin é um flavonoide extraído do cardo leiteiro e que esse apresenta grande ação antioxidante e antiinflamatória, dessa forma avaliaram a eficácia da silimarina oral na prevenção da mucosite em pacientes com câncer de cabeça e pescoço, logo ambos os pesquisadores destacam a importância dessa planta frente a redução da gravidade da radioterapia em pacientes.

O ensaio clínico de Jowkar et al. ${ }^{9}$ foi realizado em 34 pacientes com vitiligo, que é uma doença crônica na pele causada pela destruição dos melanócitos e teve como interesse mostrar o efeito da fototerapia oral da silimarina neste tratamento, com isso mostrou que provavelmente a silimarina seja uma boa alternativa para o tratamento desses pacientes, em contrapartida os efeitos hepatoprotetores dessa planta ganham grande destaque. Em sua pesquisa Barbosa et al. ${ }^{7}$ afirmam que a silimarina é conhecida tradicionalmente pelo seu efeito hepatoprotetor, mas com estudos recentes têm proposto uma nova atividade farmacológica, promover a lactogênese durante a gravidez e amamentação e tiveram como resultados testados em modelo animal a segurança de seu uso durante esse período.

Pesquisas frente às propriedades antioxidantes dessa planta tem crescido bastante, enfatizando o estudo realizado por Grant et al. ${ }^{8}$ que verificaram as propriedades antioxidantes e a eficácia da silimarina em crianças e adultos com tricotilomania, com isso um grupo com vinte indivíduos com tricotilomania passou por doze semanas recebendo dosagem do cardo leiteiro duas vezes ao dia e obtiveram como resultados que o cardo leiteiro demonstrou melhorias significativas.

\section{Considerações finais}

A presente pesquisa constatou evidências de que o tratamento com silimarina e os seus constituintes são capazes de atenuar complicações diabéticas comuns em vários órgãos, bem como enfatizaram as propriedades antioxidantes, anti-inflamatórios, antiapoptóticos dose-dependentes e modificadores de transportadores celulares presentes nessa planta, logo a silimarina apresenta importantes propriedades terapêuticas para as mais diversas enfermidades sendo necessários novos estudos aprofundados frente a essas propriedades. Logo foi visto também que há uma escassez de pesquisas sobre a domesticação e investigação das propriedades farmacológicas desta planta.

\section{Referências}

1. DAVID, Renata Boscaini; BELLO, Gabriela Brenner. Prescrição de fitoterapia por nutricionistas em farmácias magistrais. Braspen J, v. 32, n. 3, p. 288-292, 2017.

2. CACCIA-BAVA, Maria do Carmo Gullaci et al., Disponibilidade de medicamentos fitoterápicos e plantas medicinais nas unidades de atenção básica do Estado de São Paulo: resultados do Programa Nacional de Melhoria do Acesso e da Qualidade da Atenção Básica (PMAQ). Ciência \& Saúde Coletiva, v. 22, p. 16511659, 2017.

3. OLIVEIRA, Vinicius Bednarczuk. Conhecimentos e uso de plantas medicinais por usuários de unidades básicas de saúde na região de colombo, PR. Revista Brasileira de Ciências das Saúde, v. 22, n. 1, p. 57-64, 2018.

$4 . \quad J U N I O R$, Hernani Pinto de Lemos; LEMOS, André Luis Alves. Silimarina, Disponível em: $\quad<$ http://files.bvs.br/upload/S/1413- 
9979/2012/v17n1/a2842.pdf>. Acesso em: 06 fev. 2020.

DOS SANTOS, Daniel Sousa; RODRIGUES, Mayara Mikelle Farias. Atividades farmacológicas dos flavonoides: um estudo de revisão. Estação Científica (UNIFAP), v. 7, n. 3, p. 29-35, 2017.

6. COSTA, Ingrid Andresa Fernandes; DE SOUSA OLIVEIRA, Fernando. FÁRMACOS HEPATOTÓXICOS E HEPATOPROTETORES: UMA REVISÃO DE LITERATURA. JournalofBiology\&PharmacyandAgricultural Management, v. 17, n. 1, 2020.

7. BARBOSA, Caio Cesar et al. Silymarin administration during pregnancy and breastfeeding: evaluation of initial development and adult behavior of mice. Neurotoxicology, v. 78, p. 64-70, 2020.

8. GRANT, Jon E.; REDDEN, Sarah A.; CHAMBERLAIN, Samuel R. Milk Thistle Treatment of Children and Adults with Trichotillomania: A Double-Blind, Placebo-Controlled, Cross-Over Negative Study. Journal of clinical psychopharmacology, v. 39, n. 2, p. 129, 2019.

9. JOWKAR, Farideh; GODARZI, Hamid; PARVIZI, Mohammad Mahdi. Can we consider silymarin as a treatment option for vitiligo? A double-blind controlled randomized clinical trial of phototherapy plus oral Silybum marianum product versus phototherapy alone. Journalofdermatologicaltreatment, 2019.

10. EBRAHIMPOUR-KOUJAN, Soraiya et al. Lower glycemic indices and lipid profile among type 2 diabetes mellitus patients who received novel dose of Silybum marianum (L.) Gaertn.(silymarin) extract supplement: A Tripleblinded randomized controlled clinical trial. Phytomedicine, v. 44, p. 39-44, 2018.

11. KARBASFOROOSHAN, Hedyieh et al. Topical silymarin administration for prevention of acute radiodermatitis in breast cancer patients: $A$ randomized, double-blind, placebo-controlled clinical trial. Phytotherapyresearch, v. 33, n. 2, p. 379-386, 2019.

12. KAYEDPOOR, Parvin et al. Antiinflammatory Effect of Silymarin on Ovarian Immunohistochemical Localization of TNF- $\alpha$ Associated with Systemic Inflammation in Polycystic Ovarian Syndrome. InternationalJournalofMorphology, v. 35, n. 2, 2017.

13. . ELYASI, Sepideh et al. Effect of oral silymarin administration on prevention of radiotherapy induced mucositis: A randomized, double-blinded, placebo-controlled clinical trial. Phytotherapy Research, v. 30, n. 11, p. 18791885, 2016.

14. SOTO, C. et al. Effect of silymarin on kidneys of rats suffering from alloxan-induced diabetes mellitus. Phytomedicine, v. 17, n. 14, p. 1090-1094, 2010. 
Endereço para Correspondência

Antonio Rafael da Silva

Rua Ratisbona no 32 Apto 302, Centro, Crato-CE.

CEP: $63100-140$

E-mail: raphaelsilvha@gmail.com

Recebido em 14/05/2021

Aprovado em 06/08/2021

Publicado em 27/09/2021 